\title{
Evaluation of an Alternative Depression Therapy Using Bioresonance
}

\author{
Daniela Muresan', Andreea Salcudean², Bogdan Viorel Barbu¹, Cristina Raluca Bodo³, \\ Iosif Gaboș Grecu¹ \\ 1 "George Emil Palade" University of Medicine, Pharmacy, Science and Technology, Târgu Mureș, Romania \\ 2 Department of Ethics and Social Sciences, "George Emil Palade" University of Medicine, Pharmacy, Science and Technology, \\ Târgu Mureș, Romania \\ 3 Psychiatric Clinic, Mureș County Hospital, Târgu Mureș, Romania
}

\section{CORRESPONDENCE}

\section{Andreea Salcudean}

Str. Gheorghe Marinescu nr. 38

540142 Târgu Mureș, Romania

Tel: +40 755690587

E-mail: andreea.salcudean@umfst.ro

\section{ARTICLE HISTORY}

Received: March 7, 2021

Accepted: March 23, 2021
Daniela Muresan • Str. Gheorghe Marinescu nr. 38 540142 Târgu Mureș, Romania. Tel: +40 265215551 E-mail: daniela.muresan@insp.gov.ro

Bogdan Viorel Barbu • Str. Gheorghe Marinescu nr. 38, 540142 Târgu Mureș, Romania. Tel: +40 265215 551, E-mail: barbu.b@gmail.com

Cristina Raluca Bodo • Str. Gheorghe Marinescu nr. 38, 540142 Târgu Mureș, Romania. Tel: +40 265215 551, E-mail: cristina.bodo@gmail.com

losif Gaboș Grecu • Str. Gheorghe Marinescu nr. 38 540142 Târgu Mureș, Romania. Tel: +40 265215551 E-mail: iosif.gabos-grecu@umfst.ro

\begin{abstract}
Background: Bioresonance therapy is a holistic physical method used in the treatment of various diseases. Aim of the study: The aim of the present paper was to evaluate the effect of bioresonance therapy in patients with recurrent major depressive disorder and with mild or moderate depressive episodes, respectively, without any other pharmacological, psychological, or other type of treatment. Methods: We selected 40 patients diagnosed with recurrent depressive disorder and bioresonance therapy as the only treatment. Assessment of the level of depression was achieved by measuring the biorhythm with the bioresonance device and by applying the Hamilton Depression Inventory questionnaire, with 17 items, at the beginning and the end of five weeks of treatment. Results: We identified a statistically significant decrease in depression levels as quantified by the HAM-D 17 scale, between the initial and final phase of therapy ( $p=$ 0.0001). Conclusions: Our results suggest that bioresonance therapy can decrease the level of depression in patients with recurrent depressive disorder, with mild and moderate depressive episodes.
\end{abstract}

Keywords: bioresonance therapy, depression, alternative medicine, low-intensity current

\section{INTRODUCTION}

Bioresonance therapy is a holistic physical method used in the treatment of various diseases. ${ }^{1}$ The Manual of Diagnosis and Statistical Classification of Mental Disorders V stipulates that major depressive disorder presents distinct episodes lasting at least two weeks, involving obvious changes in affect, cognition, and neurovegetative functions, with inter-episodic remissions. This negative state causes suffering, significantly damaging the area of functioning in the professional or social field of the individual. ${ }^{2}$

More than 350 million people worldwide suffer from depression. ${ }^{3}$ Depression is more common among women than men. Every year, 800,000 people die as a result of suicide. ${ }^{4}$ In Europe, one in 20 people suffers from depression, and one 
in four will go through a depressive episode at some point in life. According to the World Health Organization, in Romania, about $5 \%$ of the population suffers from depression. This condition has led to 163,836 years of life lived with disabilities, representing $7.9 \%$ of the total years of life lived with disabilities in the Romanian population. ${ }^{5}$

Electroconvulsive therapy (ECT) is among the first treatments introduced in psychiatry 80 years ago and the only one still in use. The induction of a generalized controlled tonic-clonic seizure is caused by the administration of a small amount of electricity, being effective in treating depression without worsening cognitive functions. ${ }^{6}$

We aimed to evaluate if a personalized bioresonance therapy for patients with recurrent major depressive disorder and with mild or moderate depressive episode, respectively, can decrease the level of depression without the use of any other pharmacological or psychological treatment. The level of depression was evaluated within 2 months after receiving the first bioresonance treatment, using the Hamilton Depression Inventory questionnaire, which includes 17 items.

\section{METHODS}

We selected patients diagnosed by a psychiatrist with recurrent major depressive disorder and with mild to moderate depression, according to the criteria listed in the Diagnostic Manual of Mental Disorders DSM-V.

The patients were selected from Psychiatry Clinic I of the Mureș County Clinical Hospital in Târgu Mureș, Romania and from a specialized outpatient clinic.

The study was retrospective and was conducted between October 2017 and October 2018. Exclusion criteria were represented by the presence of pacemakers, pregnancy, and patients with suicide attempts.

By applying the bioresonance therapy, we aimed to decrease the level of depression, evaluated with HAM-D 17, from the first to the fifth therapy session, within a maximum period of 2 months.

Bioresonance therapy consisted of connecting the patient to the Mora Nova medical device, using two-dimensional electrodes for the hands and feet. At the beginning of therapy the biorhythm is measured, the device automatically displaying the conductance between the left hand and right hand, the left hand and left foot, the right hand and right foot, and the left foot and right foot, respectively. During each treatment session, the device automatically records the values of the eight quadrants, obtained at the first and second measurements. During the measurement of values, the device emits sounds of different intensity, depending on the values obtained. ${ }^{7}$
Bioresonance therapy used the biorhythm program, which allowed us to assess whether the patient was normoreactive, hyporeactive, or hyperreactive, according to the energy level and the potential to respond to the actual applied therapy, observing whether there was a change in body polarity and whether there was a response to the applied therapy, using a low intensity current. During the therapy session, it is recommended that the patient enters a state of relaxation, without being distracted or interacting with other people, the therapist being the only person who can be around him. The duration of a session was about 20 minutes, with a frequency of one session per week.

The applied therapy was personalized for each patient, according to the energy level of the treated person, incorporating high and low potentials, in line with the patient's needs. Through the biorhythm program and the 163 Yin Yang balancing with the LU-7, SI-3 program, respectively, the therapeutic method was selected from the device software, adjusting and controlling the patient's own oscillating information, as well as the ability to adjust in order to initiate a targeted healing process. During the 163 Yin Yang balancing with the LU-7, SI-3 program, the additional bipolar electrode Blue Mag S was used, which was provided with eight magnets and a blue light, thus regulating the magnetic field by making circular movements to the head for 43 seconds, avoiding the eye area.

Biorhythms were obtained from coupling the electromagnetic oscillators and the division of resistance frequencies, getting fast oscillations and one or two frequencies, which were quite slow. Overlapping the heartbeat by coupling two electric oscillators can speed up the frequencies of the oscillator. During therapy, the patient did not carry an electronic device, metals in contact with the skin, or mechanical devices. Before and after each treatment session, the electrodes were cleaned with $70^{\circ}$ alcohol. ${ }^{8}$

The Mora Nova device is a modern, transceiver electromagnetic device, which must be connected to a source of electricity; it is equipped with a backup battery, with a frequency between $0.1 \mathrm{~Hz}$ and $480,000 \mathrm{~Hz}$, and a frequency filter from $1 \mathrm{~Hz}$ up to $500,000 \mathrm{~Hz} .^{9}$

By measuring the biorhythm at each treatment session before and after the application of the therapeutic program, we evaluated if the patient had any specific reaction to the applied electronic therapy. This was achieved by displaying the measurements on the device screen, showing the mental state in which the patient was before and at the end of each treatment. The interpretation of the energetic situation and of the degree of depression at the moment of examination, respectively, was achieved in relation to the physiological values framed between $80-85 \mathrm{~Hz}$, values cor- 
responding to a healthy patient. If the energy values were below 80 , the patient was considered hyporeactive, with inhibited depression or agitated-anxiety depressive syndrome, and if the values were above 85 , he was considered hyperreactive, indicating an agitated depression or inhibited-anxiety depressive syndrome. ${ }^{7}$

The evaluation of the depression was performed with HAM-D 17 at the beginning and at the end of the five weeks of bioresonance treatment, by a clinical psychologist.

HAM-D 17 is a questionnaire composed of questions rated with $0-2$ or $0-4$ points, 4 being the most severe. The total score of the HAM-D 17 scale varies between 0 and 52 points. The scale measures individual depressive symptoms and their general severity, the final score indicating the level of depression: $>25$ indicates severe depression, 18-24 moderate depression, 8-17 mild depression, and a score $<7$ is considered normal. Statistical indicators were calculated using Microsoft Excel, Google Docs, and GraphPad. Differences between average values were compared using Student's t test, and session results were compared using ANOVA. A p value $<0.05$ was considered to be statistically significant.

Written consent was obtained from participants after they were informed about the study and its implications. Consent was also obtained from the appropriate Romanian authorities. Data protection was ensured. The study was approved by the Institutional Ethics Committee of the Mureș County Hospital from Târgu Mureș (approval number $16462 / 16.10 .2017)$. The study complied with the Declaration of Helsinki.

\section{RESULTS}

The study group consisted of 40 patients aged between 19 and 71 years, and $77.50 \%(\mathrm{n}=31)$ were females. Regarding the patients' marital status, $50 \%$ were married, $30 \%$ divorced, and $20 \%$ single. Regarding the education level, $30 \%$ had high school education, $12.5 \%$ college education, $5 \%$ vocational school, and $52.5 \%$ higher education. Thirty-five per cent of patients were from rural areas and $65 \%$ from urban areas.

Five men were diagnosed with a mild depressive episode and 4 men with a moderate depressive episode. Seventeen women had a mild depressive episode, and 14 women presented with a moderate depressive episode.

The results obtained with the HAM-D 17 questionnaire are shown in Figure 1. The average level of depression diminished significantly, with a reduction of 3.10 points ( $p$ $=0.0001)$. The results obtained before and after the treatment are presented in Table 1.
TABLE 1. Descriptive statistics of the initial and final Hamilton Rating Scale

\begin{tabular}{lccc}
\hline & \multicolumn{3}{c}{ Hamilton Rating Scale scores } \\
\cline { 2 - 4 } & Initial & Final & Difference \\
\hline Minimum & 12.00 & 8.00 & -6.00 \\
25\% Percentile & 14.00 & 12.00 & -4.00 \\
Median & 16.50 & 14.00 & -2.50 \\
75\% Percentile & 19.00 & 16.00 & -2.00 \\
Maximum & 24.00 & 21.00 & -2.00 \\
Mean & 16.90 & 13.80 & -3.10 \\
SD & 3.23 & 3.18 & 1.27 \\
\hline
\end{tabular}

Table 2 presents the patients' distribution according to their energy level and their evolution following the application of bioresonance therapy. The comparison was made during each session before and after therapy, but also to highlight the global effect of therapy between the first and the fifth session.

After five sessions of bioresonance therapy, the results showed that in the initial phase, one person went from normal values to hyperreactive values, and in the real phase, 14 patients with depression, of which 11 hyporeactive and 3 hyperreactive, registered normal values (Table 3 ).

The distribution of hyperreactive, hyporeactive, and normal subjects according to biorhythm is shown in Figure 2. There were no statistically significant differences in the type of biorhythm between the initial assessment and after each bioresonance session ( $p>0.05$ ) (Figure 2 ).

The proportion of hyperreactive patients improved significantly between the first and the fifth session of bioreso-

\section{$p=0.0001$}

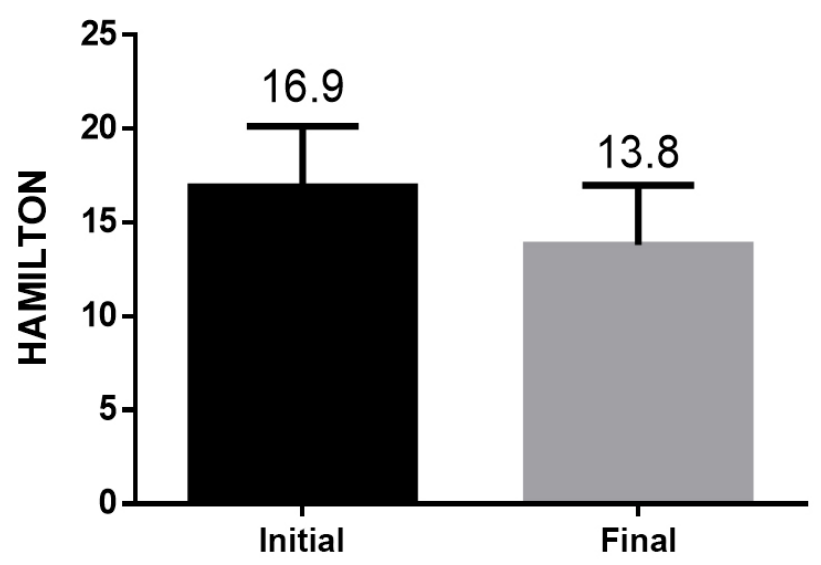

FIGURE 1. Comparison of the Hamilton Depression Scale between the initial and final session for the total study population 
TABLE 2. Evolution and comparison of the hyperreactive, hyporeactive and normal persons for each session, according to biorhythm and depression score, evaluated at the beginning and ending of each therapy session

\begin{tabular}{lcccccccccc}
\hline & \multicolumn{2}{c}{ Session } & \multicolumn{2}{c}{ Session 2 } & \multicolumn{2}{c}{ Session 3 } & \multicolumn{2}{c}{ Session 4 } & \multicolumn{2}{c}{ Session 5 } \\
\cline { 2 - 12 } & Initial & Final & Initial & Final & Initial & Final & Initial & Final & Initial & Final \\
\hline Hyperreactive & 9 & 9 & 14 & 7 & 12 & 7 & 13 & 7 & 10 & 6 \\
Hyporeactive & 19 & 26 & 18 & 23 & 19 & 25 & 21 & 22 & 19 & 15 \\
Normal & 12 & 5 & 8 & 10 & 9 & 8 & 6 & 11 & 11 & 19 \\
Total & 40 & 40 & 40 & 40 & 40 & 40 & 40 & 40 & 40 & 40 \\
P value & \multicolumn{2}{c}{0.15} & \multicolumn{2}{c}{0.23} & & 0.33 & & 0.20 & & 0.15 \\
\hline
\end{tabular}

TABLE 3. Comparison between the first and the last bioresonance session for minimum, maximum, and mean values

\begin{tabular}{|c|c|c|c|c|c|c|c|c|c|c|c|c|}
\hline \multirow[b]{3}{*}{ Bioresonance session } & \multicolumn{4}{|c|}{ Session 1} & \multicolumn{4}{|c|}{ Session 2} & \multicolumn{4}{|c|}{ Session 3} \\
\hline & \multicolumn{2}{|c|}{ Initial } & \multicolumn{2}{|c|}{ Final } & \multicolumn{2}{|c|}{ Initial } & \multicolumn{2}{|c|}{ Final } & \multicolumn{2}{|c|}{ Initial } & \multicolumn{2}{|c|}{ Final } \\
\hline & s1 & S5 & s1 & S5 & s1 & S5 & s1 & S5 & S1 & S5 & s1 & S5 \\
\hline Mean & 69.9 & 73.4 & 68.9 & 73.8 & 86.4 & 86.5 & 85.5 & 86 & 78.4 & 79.9 & 77.1 & 79.8 \\
\hline SD & 11.4 & 12.0 & 11.1 & 9.07 & 11.6 & 6.5 & 9.9 & 6.4 & 10.7 & 8.18 & 9.79 & 6.98 \\
\hline Median & 69 & 75.5 & 69.5 & 75.5 & 91 & 88 & 88.5 & 86 & 80.3 & 81.2 & 77.1 & 81.3 \\
\hline Min & 47 & 26 & 43 & 47 & 56 & 74 & 58 & 69 & 51.4 & 53.5 & 50.8 & 62.3 \\
\hline Max & 91 & 92 & 87 & 90 & 100 & 97 & 99 & 98 & 94.8 & 93 & 93 & 93.6 \\
\hline
\end{tabular}

nance therapy, from $7.5 \%$ to $33.34 \%$ ( $p=0.002)$. Similarly, in case of hyporeactive patients, there was a significant improvement in depression levels between the first and fifth bioresonance session, from $27.5 \%$ to $42.30 \%$ ( $p=0.001)$.

\section{DISCUSSIONS}

In this study, we aimed to analyze whether bioresonance therapy can decrease the level of discomfort, evaluated

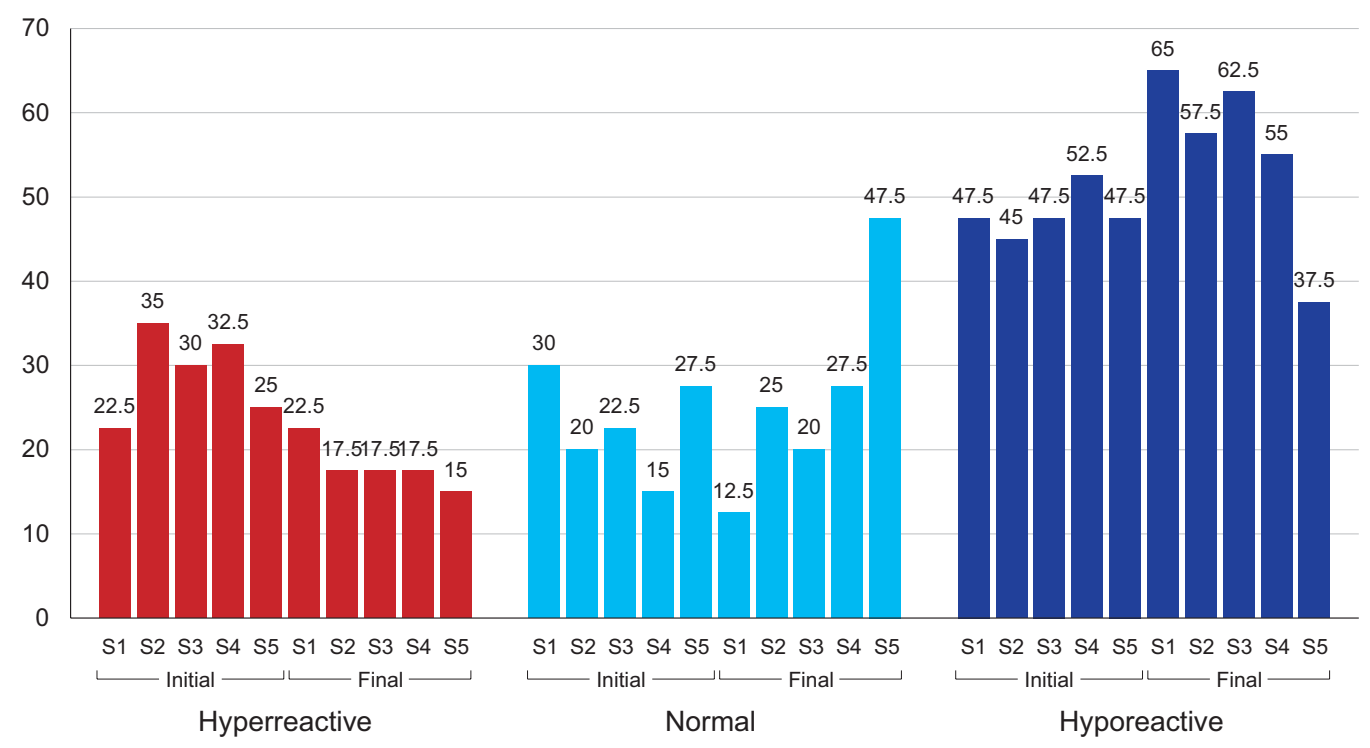

FIGURE 2. Distribution of hyperreactive, hyporeactive, and normal patients, according to biorhythm at each bioresonance session 
with the HAM-D 17 scale, for patients diagnosed with depressive disorders.

Bioresonance therapy was offered as an alternative therapeutic solution according to the patients' preferences and also to patients who had been unresponsive to pharmacological and psychological therapies.

Scientific research conducted in recent years has confirmed the existence of a bioelectromagnetic field in the human body. ${ }^{8}$ The specialized literature offers examples when bioresonance therapy was successful in various pathologies. To our knowledge, there are no studies related to the efficacy of bioresonance therapy for patients with recurrent major depressive disorder, or with a mild or moderate depressive episode.

In 2019, a study conducted in Greece, which included 311 patients, women and men aged between 2 and 76 years, found that bioresonance was effective in treating lacrimation, runny nose, and respiratory and gastrointestinal disorders. After applying bioresonance therapy for 12 months, $90 \%$ of the patients no longer showed symptoms. ${ }^{1}$ Another study had proved the efficacy of bioresonance therapy for elderly patients with urinary incontinence..$^{9}$ A study from China on subjects with trigeminal neuralgia illustrated the hypothesis of bioresonance, stating that when the frequency of vibrations of a structure surrounding the trigeminal nerve becomes close to its natural frequency, resonance of the trigeminal nerve occurs. Bioresonance can damage trigeminal nerve fibers and lead to abnormal impulse transmission which can eventually lead to facial pain. ${ }^{10}$

Biofeedback therapy has been shown to be effective in reducing psychological symptoms in patients with anxiety. This suggests that biofeedback therapy may be beneficial for patients with chronic pain, along with high levels of anxiety or associated depression. ${ }^{11}$

Bioresonance therapy has no side effects, is painless and noninvasive, giving patients with depression a state of well-being, helping them to overcome the emotional and mental blockage to which they are anchored. We tested the efficacy of a personalized therapeutic method based on bioresonance, and we succeeded to obtain a statistically significant reduction in the level of depression of $40 \mathrm{pa}-$ tients. Further studies on larger populations are required for validation of this novel therapeutic method in patients with depressive disorders.

\section{CONCLUSIONS}

Our study aimed to evaluate if bioresonance therapy can improve symptoms in patients with depressive disorders. There was a significant reduction in depression levels according to the HAM-D 17 scale between the first assessment and after the fifth bioresonance therapeutic session. Bioresonance therapy may represent an alternative treatment solution for patients with recurrent major depressive disorder, with mild and moderate depressive episodes.

\section{CONFLICT OF INTEREST}

Nothing to disclose.

\section{REFERENCES}

1. Krakos P, Grigorios T, Theodoros K, Theodoros L. The effectiveness of bioresonance method on human health. The Open Epidemiology Journal. 2019;8:1-8.

2. Goia MC, Popp CG, Botez CA. DSM-5: manual de diagnostic şi clasificare statistică a tulburărilor mintale. Bucureşti: Editura Medicală Callisto, 2016; p. 155, 161-162.

3. Marcus M, Yasamy MT, van Ommeren M. Depression: A Global Public Health Concern. World Health Organization Paper on Depression. 2012;68.

4. Spencer J, Abate D, Abate H, et al. Global, regional, and national incidence, prevalence, and years lived with disability for 354 diseases and injuries for 195 countries and territories, 1990-2017: a systematic analysis for the Global Burden of Disease Study 2017. The Lancet. 2018;392:1789-1858.

5. World Health Organization. Depression and Other Common Mental Disorders. Global Health Estimates. Geneva. 2017. Available at: https:// www.who.int/mental_health/management/depression/en/

6. Kaliora SC, Zervas IM, Papadimitriou GN. Electroconvulsive therapy: 80 years of use in psychiatry. Psychiatriki. 2018;29:291.

7. Chiriță V, Papari A, Chiriță R (eds). Tratat de psihiatrie, Vol I. Constanța: Editura Fundației "Andrei Șaguna”, 2009; p. 346.

8. Minser OP, Potiazhenko MM, Nevoit GV. Energy harvesting for the implantable biomedical devices: issues and challenges. Wiad Lek. 2019;72:1117-1121.

9. Cherniack EP. Biofeedback and other therapies for the treatment of urinary incontinence in the elderly. Altern Med Rev. 2006;11:224-231.

10. Jia DZ, Li G. Bioresonance hypothesis: a new mechanism on the pathogenesis of trigeminal neuralgia. Med Hypotheses. 2010;74:505-507.

11. Rotkis LN, Abelon R, Collette $C$. The effect of biofeedback therapy on depression and anxiety in the pediatric and adolescent when used to treat migraines, chronic headaches and chronic abdominal pain Breuner. Journal of Adolescent Health. 2014;54:S42. 